
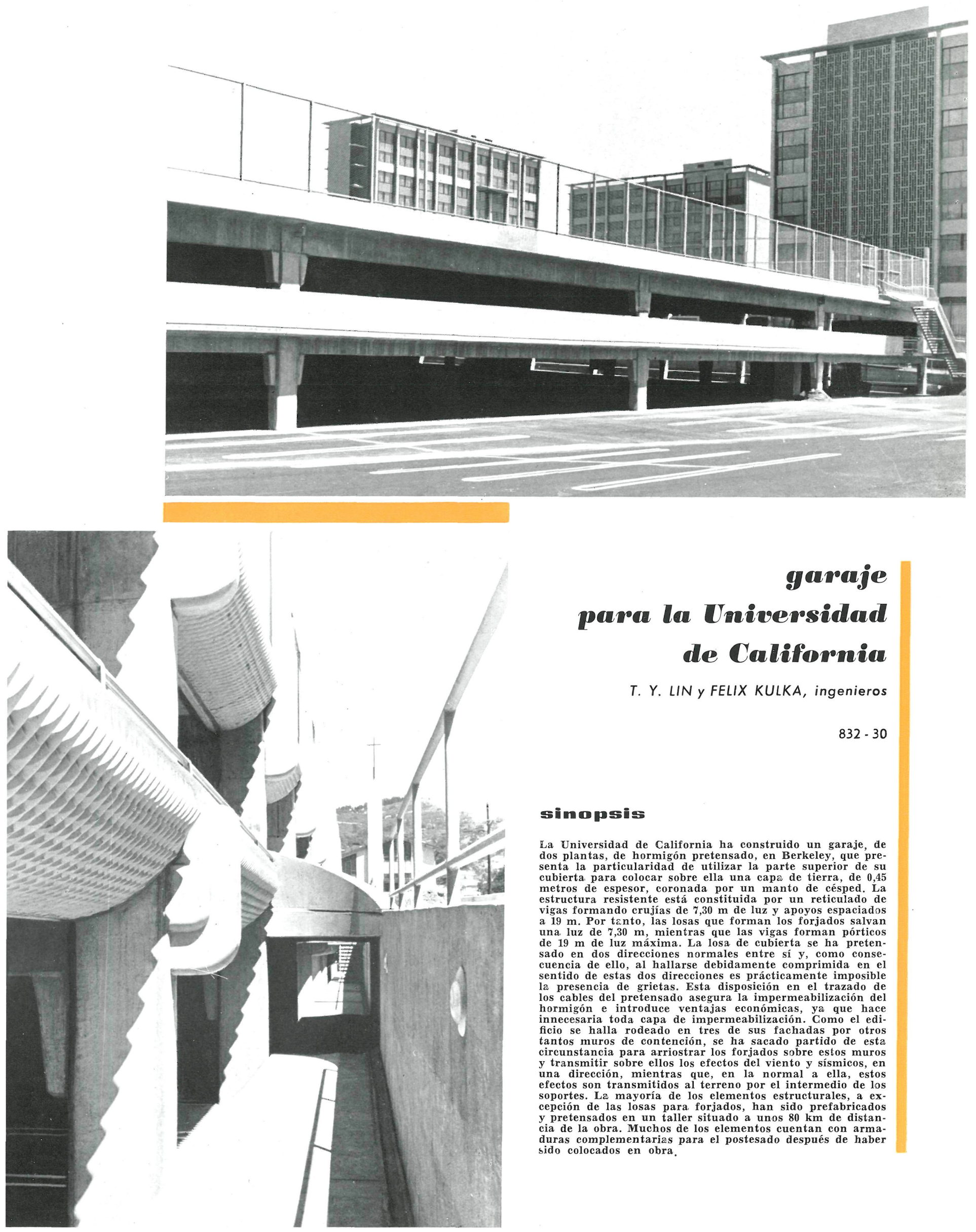

\title{
garaje \\ para la Universidad de California
}

T. Y. LIN y FELIX KULKA, ingenieros

$832 \cdot 30$

\section{sinopsis}

La Universidad de California ha construido un garaje, de dos plantas, de hormigón pretensado, en Berkeley, que presenta la particularidad de utilizar la parte superior de su cubierta para colocar sobre ella una capa de tierra, de 0,45 metros de espesor, coronada por un manto de césped. La vicas formando a 19 Por tonto, las losas que forman los forjados sados una luz de $730 \mathrm{~m}$ mientras que las vigas forman pórticos de $19 \mathrm{~m}$ de luz máxima. Ia losa de cubierta se ha pretensado en dos direcciones normales entre sí y, como consecuencia de ello, al hallarse debidamente comprimida en el sentido de estas dos direcciones es prácticamente imposible la presencia de grietas. Esta disposición en el trazado de los cables del pretensado asegura la impermeabilización del hormigón e introduce ventajas económicas, ya que hace innecesaria toda capa de impermeabilizacion. Como el edificio se halla rodeado en tres de sus fachadas por otros tantos muros de contencion, se ha sacado partido de esta circunstancia para arriostrar los forjados sobre estos muros y transmitir sobre ellos los efectos del viento y sísmicos, en una direccion, mientras que, en la normal a ella, estos efectos son transmitidos al terreno por el intermedio de los cepción. de las losas para forjados, han sido prefabric $y$ pretensados en un taller situado a unos $80 \mathrm{~km}$ de distany pre duras complementarias para el postesado después de haber sido colocados en obra. 


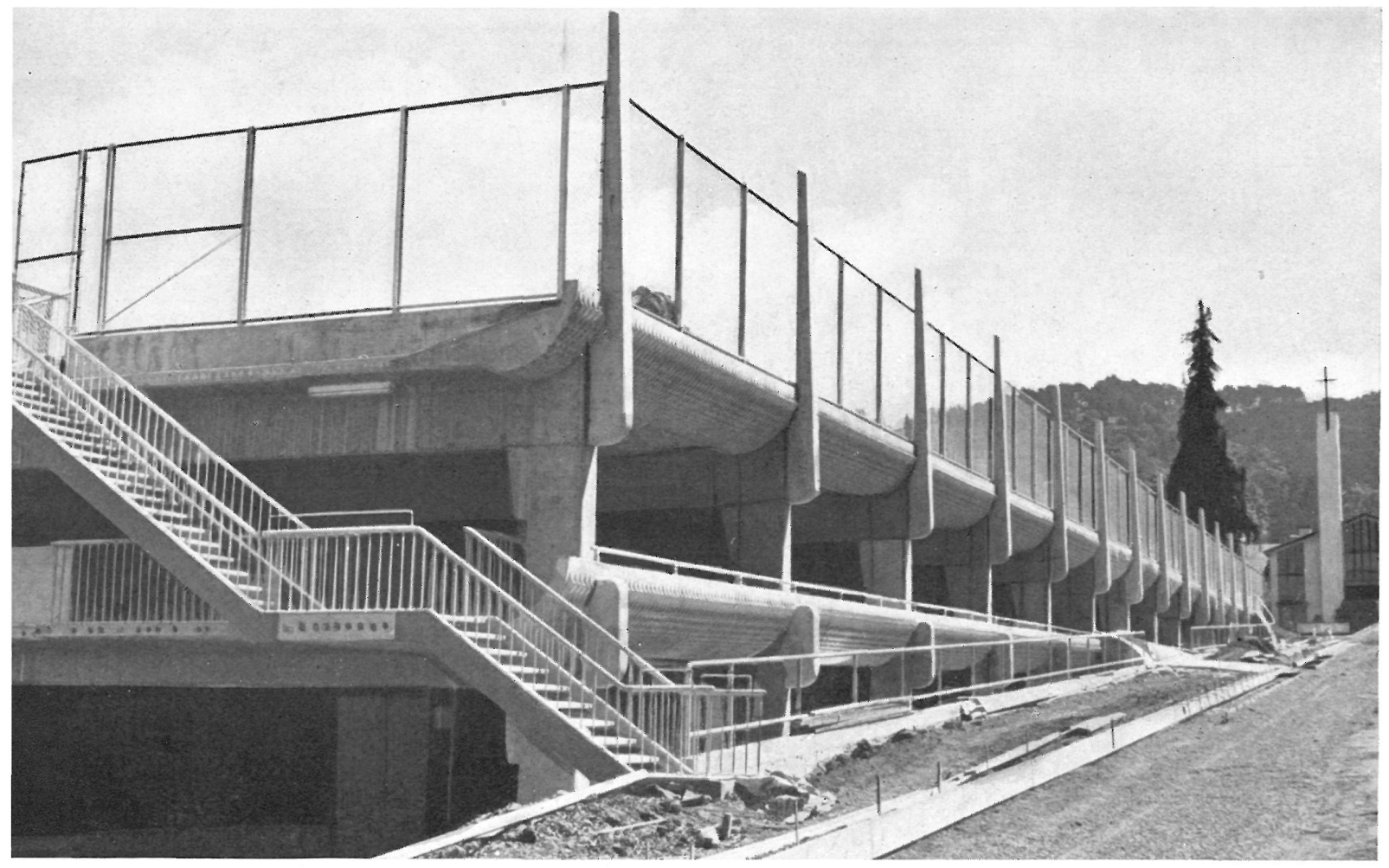

Recientemente se ha terminado un garaje, con estructura pretensada, para la Universidad de California, en Berkeley. Se ha proyectado teniendo en cuenta todos los avances realizados en el campo del hormigón pretensado, consiguiéndose así una construcción de utilidad máxima y mínimo coste.

Aunque los conceptos esenciales de esta estructura han sido aplicados con éxito en más de otras 50 similares, proyectadas por la misma firma, la incorporación de un "campus» en este garaje es de una notable originalidad y marca nuevos caminos.

Tiene capacidad para 650 automóviles y está situado en el centro de seis edificios, de plantas múltiples, en los que se distribuyen los estudios y habitaciones para unos 2.000 estudiantes.

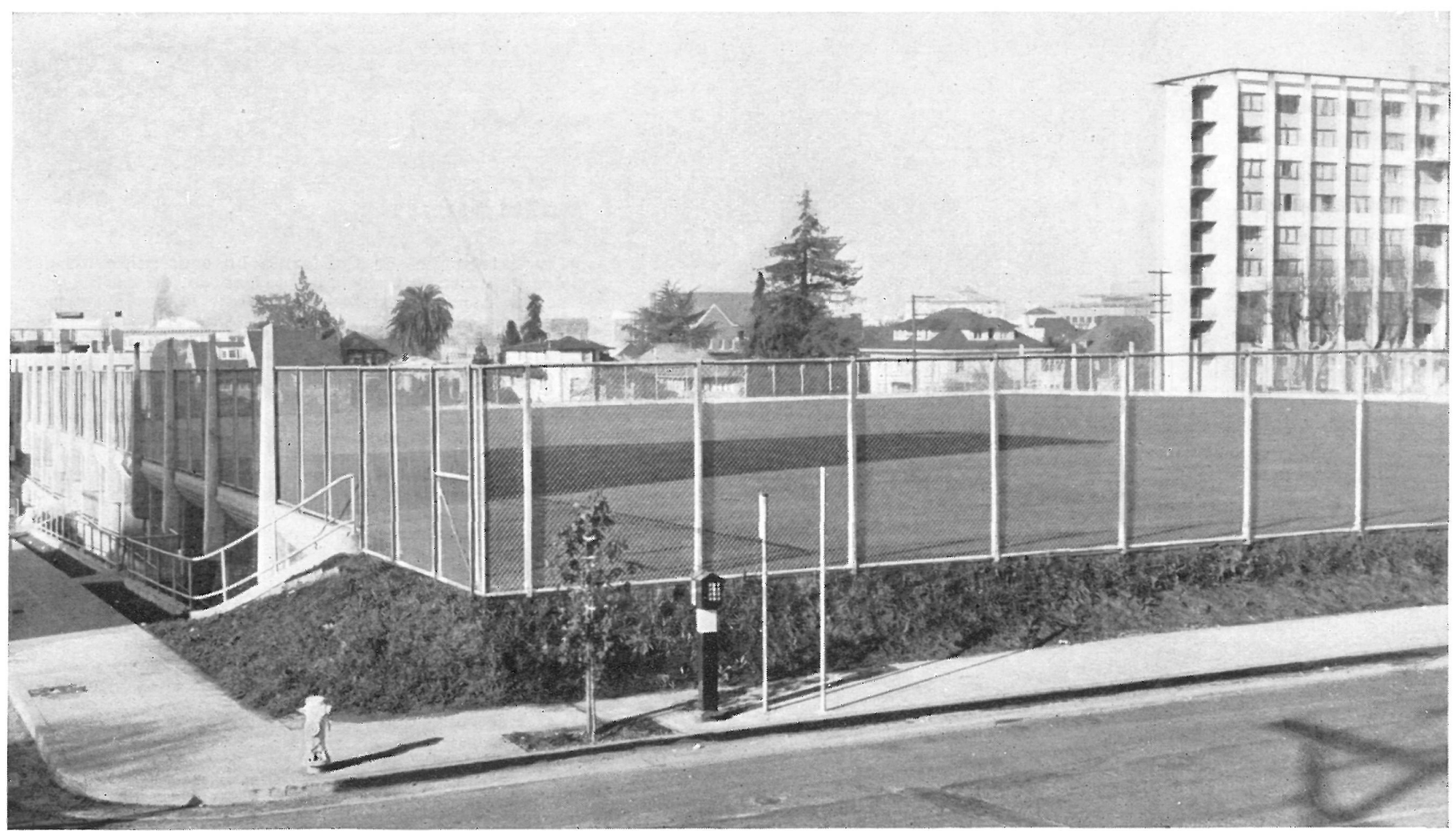




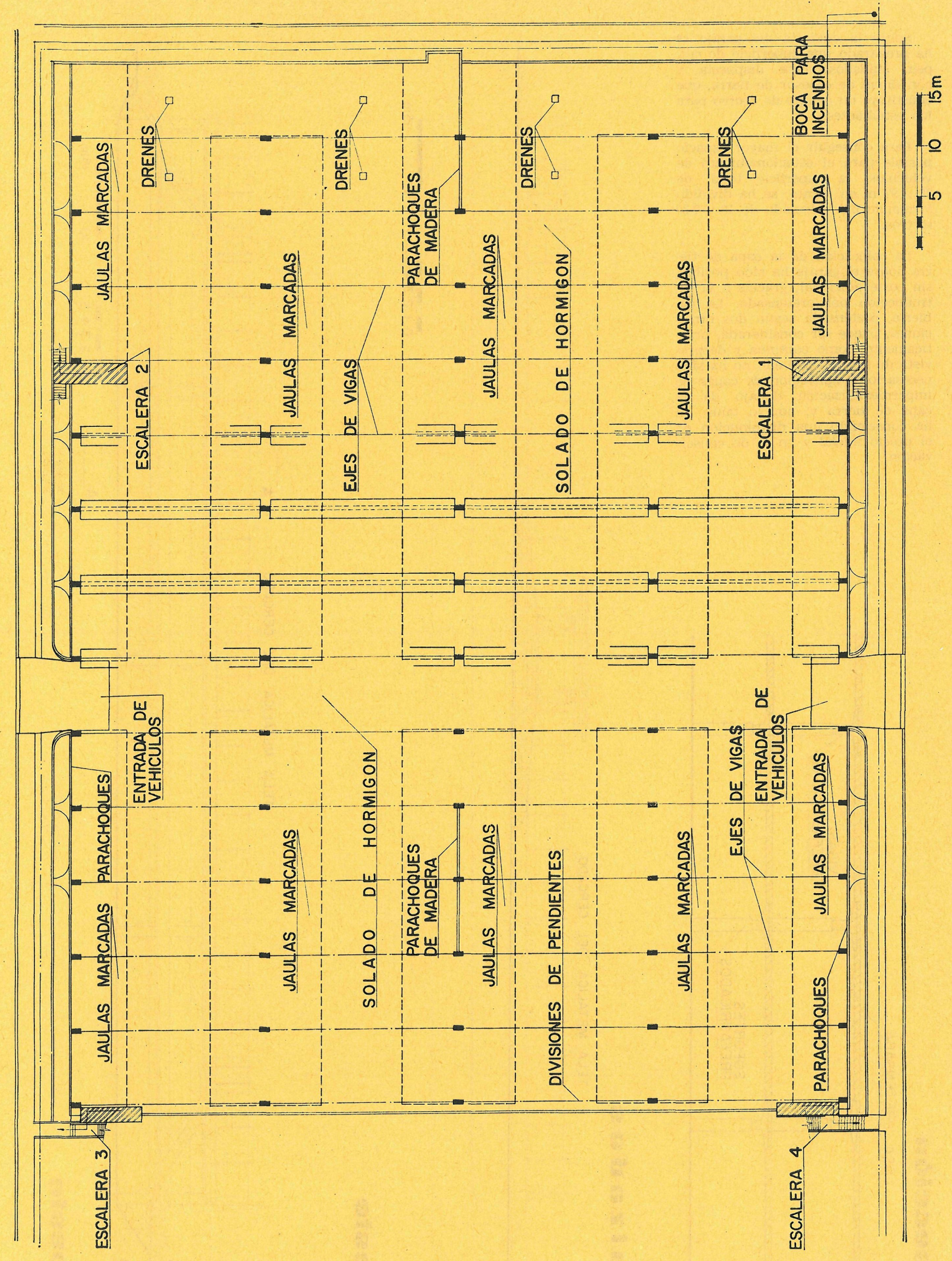

pla nta 
Sobre la cubierta del garaje se ha preparado una superficie de césped, cuidadosamente dispuesta y con $45 \mathrm{~cm}$ de espesor de tierra, que constituye un campo de recreo para los estudiantes.

Para conseguir el mayor espacio posible para el estacionamiento de vehículos, la superficie total, de $78 \times 97 \mathrm{~m}$ en planta, se ha dividido formando un reticulado de $19 \times 7,30$ metros.

La colocación de la capa de tierra sobre el garaje ha sido posible, sin coste adicional, gracias a la introducción del pretensado. La cubierta, pretensada según dos direcciones, puede ser considerada, prácticamente, como continua y desprovista de grietas; por ello no ha sido necesario la aplicación de capas de impermeabilización debajo de la capa de tierra $\mathrm{y}$, por lo tanto, se ha conseguido una disminución en el precio inicial y en el de conservación.

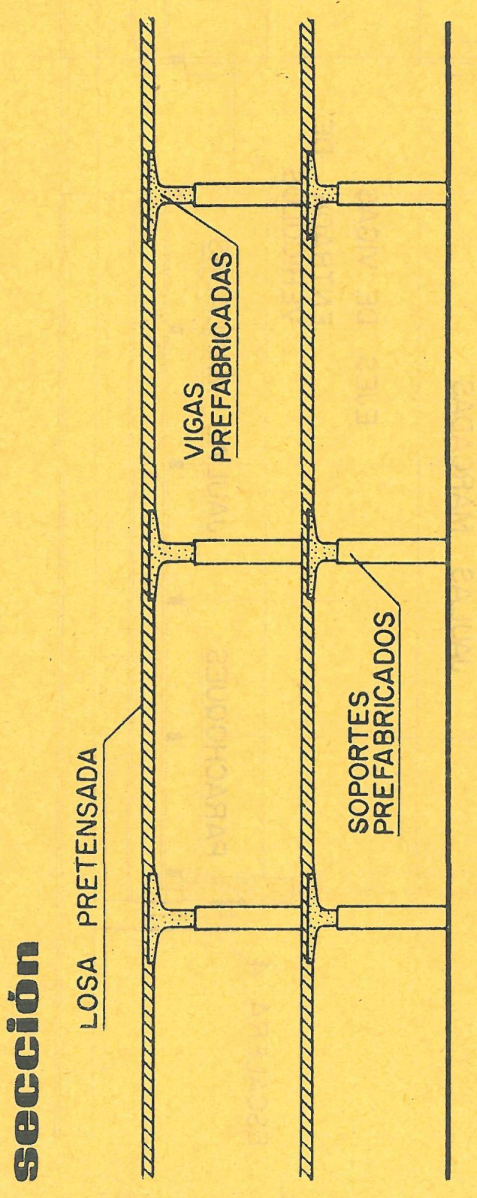

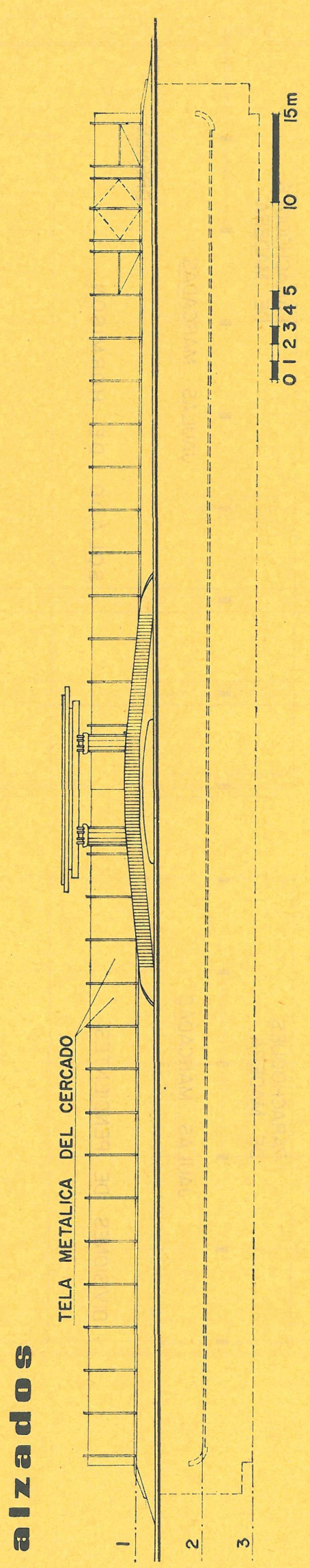

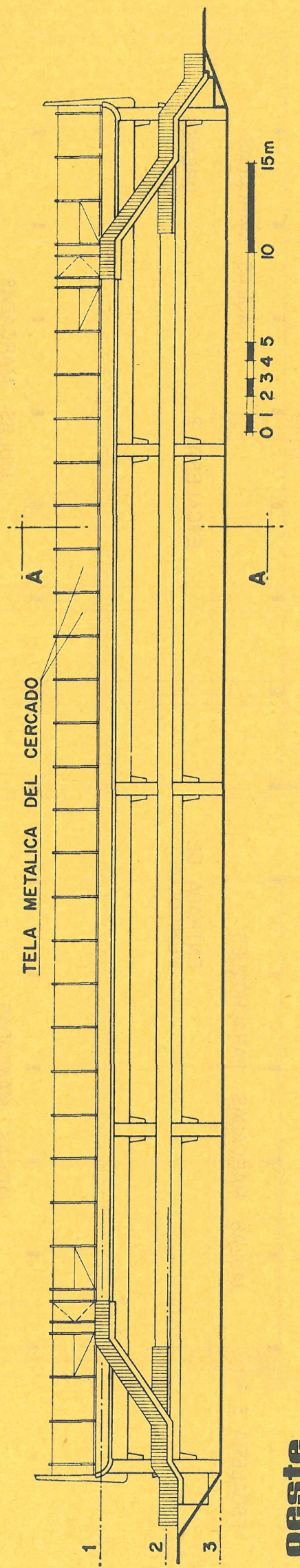


Pórticos

Losa pretensada de cubierta.

Cables del pretensado de cubierta.

Los grandes huecos de fachada dan la sensación de que el garaje se halla integrado en la naturaleza, lo cual constituye una atracción para los estudiantes.

En la apertura de curso fue habilitado este garaje para celebrar un baile estudiantil, al que asistieron más de 1.000 parejas, demostrando, de esta manera, los múltiples usos de este tipo de edificaciones.

Comparando esta estructura con otra de tipo tradicional, de hormigón armado, ha resuitado ser un 12 por 100 más económica, ya que se han podido obtener luces mucho mayores y menor número de soportes.

Gracias a una serie de combinaciones y conceptos introducidos en el proyecto, así como a nuevas técnicas constructivas, se ha podido lograr la versatilidad de la construcción con un coste reducido. Todas estas técnicas pueden resumirse en los seis siguientes apartados:

1. Transmisión simple y directa de las fuerzas en toda la estructura.

2. Una combinación entre la prefabricación y el hormigonado en obra.

3. Conjugación entre el pretensado y el postesado.

4. Armaduras de pretensado en dos direcciones y continuidad en las mismas.

5. Control de la elasticidad, plasticidad y retracción.

6. Control de flechas y deformaciones.

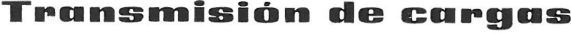

Todas las cargas verticales se transmiten a los cimientos lo más directamente posible. Las losas de los forjados salvan luces de $7,30 \mathrm{~m}$ y transmiten directamente su carga a las vigas que las soportan, espaciadas también a $7,30 \mathrm{~m}$, trasladando sus cargas a los soportes, con separación de $19 \mathrm{~m}$. La reduc ción a un mínimo del número de elementos estructurales, no solamente reduce el volumen del hormigón y mano de obra, sino que simplifica los encofrados, lo que se traduce en una reducción del coste general.

A lo largo de tres fachadas del edificio se han construido otros tantos muros de con tención, de $6 \mathrm{~m}$ de altura, que se han arrios trado a los forjados del garaje por medio de unas losas que permiten la transmisión de esfuerzos horizontales al terreno.

Los esfuerzos debidos a efectos sísmicos desarrollados en la dirección de las vigas son absorbidos por el sistema de pórticos, mientras que en la otra dirección se encargan de esta misión las losas de arriostramiento y los muros de contención anteriormente mencionados.



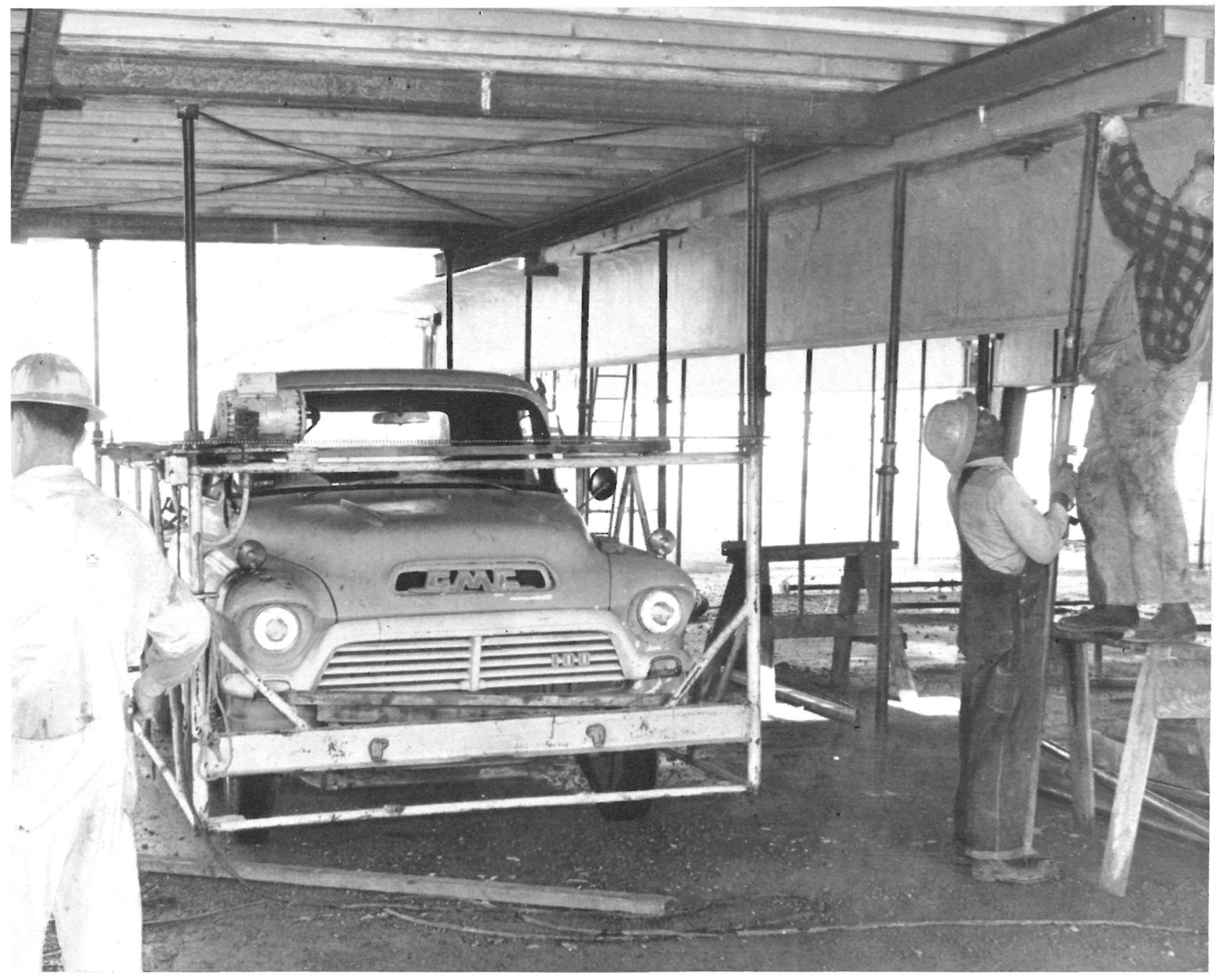

Encofrados móviles para las losas de forjados

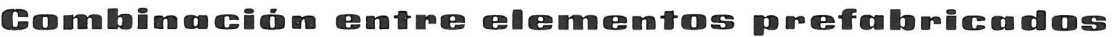 y hormigomes en obra}

Los elementos prefabricados se emplearon siempre que su grado de multiplicidad lo permitiese. La prefabricación-como es sabido-no sólo permite una economía de encofrados, sino la obtención de productos de mejor calidad $\mathrm{y}$, complementariamente, hace posible la preparación de elementos durante la cimentación de la obra. Todo ello permitió la prefabricación de soportes, con altura de dos plantas, y vigas, de $19 \mathrm{~m}$ de longitud, en el mismo taller donde se pretensaron, situado a unos $80 \mathrm{~km}$ de distancia de la obra. La totalidad de los soportes del edificio y las vigas que se extienden a lo largo de la crujía central se montaron en dos semanas, mientras que las dos filas de vigas exteriores se montaron después de un tiempo reducido.

Todas las losas que se unen a las vigas prefabricadas fueron hormigonadas en obra y dieron lugar a un aumento de la resistencia de las vigas, las cuales están provistas de alas apropiadas y tienen una especie de acartelamiento que mejora la resistencia y disminuye los efectos de flexión en las losas de forjados.

La parte curva, que se proyecta verticalmente hacia arriba en las losas de cubierta, sirve para contener la capa de tierra que constituye la zona de césped superior; en las plantas inferiores, estas formas curvadas desempeñan el oficio de soporte para los parachoques de los coches. Los paramentos vistos de esta parte de las losas tienen una textura particular: se parece mucho a la de las alcachofas, cuyas escamas constituyen una característica estética del garaje. Aunque esta forma produce una sensación aparente de dificultad, su moldeado, por el contrario, resultó relativamente sencillo, y sólo se utilizó un par de encofrados de plástico, con cuyo reempleo fue suficiente para terminar toda la obra. Los apéndices en forma de ala, acoplados a los soportes, y cuyo objeto principal es el de protezer los anclajes de los cables postesados de las vigas, fueron igualmente prefabricados en obra, utilizando encofrados de plástico. 


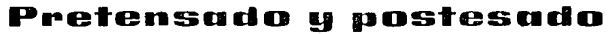

Las vigas prefabricadas se pretensaron en el taller, utilizando cables de 7 alambres de $11 \mathrm{~mm}$ de diámetro. Este pretensado no solamente ha introducido cierta economía, sino que también ha dado una excelente adherencia y mejorado la resistencia a ruptura de las vigas; sin embargo, para conseguir la continuidad y disminuir flechas se necesitó de cables complementarios postesađos en las vigas. Estos cables son del tipo Crespon y constituyen un sistema de postesado que combina una serie de alambres de $6,3 \mathrm{~mm}$ de diámetro, en cuyas extremidades se han formado, en frío, una especie de botones o esferitas.

Las vigas principales de los forjados del garaje tienen $0,58 \mathrm{~m}$ de canto y fueron pretensadas con 14 cordones y 2 cables de postesado, de 20 alambres cada uno. Las vigas de cubierta que han de sostener la capa de tierra, de $0,45 \mathrm{~m}$ de espesor, tienen $0,91 \mathrm{~m}$ de canto y se han pretensado con 14 cordones $\mathrm{y}$, además, 6 cables de postesado de 24 a 32 alambres por cable.

Las operaciones de tesado de las vigas se realizaron después de haber sido colocadas las dos filas de vigas centrales y convenientemente arriostradas.

Terminada esta primera fase de tesado, las vigas centrales se arriostraron con las exteriores, después de lo cual fueron tesadas estas últimas. En lo que respecta a las vigas de cubierta, a las operaciones de tesado siguieron las de postesado. Estas se realizaron en dos fases sucesivas: en la primera se tesaron 5 cables después se colocó la mitad de la capa de tierra que constituye la zona de césped sobre cubierta y, en la segunda fase, se tesó el último cable. En estas operaciones se permitió una pérdida elevada de rozamiento, ya que las zonas interiores requieren menos tensión que las exteriores.

El hormigonado de las losas de cubierta se ejecutó por bandas sucesivas con objeto de conseguir mayor economía y disminuir, en la medida de lo posible, el rozamiento en las operaciones de tesado. En estas operaciones, cables de $12 \mathrm{~mm}$ de diámetro, formados por 7 alambres, se iban tesando continuamente según el sistema Atlas.

\section{Tescudo y comtinuidud en dos direcciones}

El postesado de las vigas puso indirectamente en compresión, con la misma dirección que las vigas, a la losa. Ello, junto con el postesado de estas últimas, dio lugar a una compresión biaxil en las losas, obteniéndose, como resultado, prácticamente la supresión de grietas. Aunque durante el curado aparecieron varias grietas como consecuencia de la retracción, la mayor parte de ellas se cerraron después de las operaciones de postesado, y las que aún quedaban se sellaron con productos que garantizan una impermeabilización permanente.

Aunque muchas veces no resulta económica la obtención de la continuidad en dos direcciones, en este caso se logró mejorar la resistencia y rigidez. Se estimó que la continuidad mejoraba en un 10 por 100 la resistencia de las vigas y un 30 por 100 la correspondiente a las losas. Esto se consiguió sin coste adicional alguno en las losas, pero produjo cierto encarecimiento en las vigas. La continuidad de las vigas se justifica debido a la carga considerable a que se halla sometida la cubierta y, además, por la rigidez resultante de dicha continuidad, necesaria para resistir las fuerzas laterales debidas al viento y a efectos sísmicos.

\section{Bontrol de las defommenciomes elásticus, plísticas y de retraceión}

Uno de los problemas más importantes en este tipo de construcciones consiste en controlar las deforma. ciones del hormigón. De no hallar una solución propia para estas deformaciones, del conjunto de ellas pue. de derivarse: fisuración, pérdida de pretensado y disrupción de uno a otro elemento. Al emplear vigas prefabricadas y pretensadas, gran parte de la retracción, deformaciones elásticas y lentas se desarrollan antes de la vinculación estructural de dichos elementos prefabricados. El acortamiento de vigas y losas a lo largo de su longitud es, como consecuencia del postesado, relativamente reducido, ya que la compresión media en las losas resulta pequeña. Se ha previsto la presencia de algún movimiento, por lo que se han colocado algunos soportes para permitir tales movimientos sin que, por ello, se introduzcan efectos perjudiciales. 
Las operaciones de postesado, simultáneas, en dos o tres bandas, dieron lugar a un pequeño acortamiento antes de que fuera postesada la banda contigua. Estas bandas fueron separadas de sus soportes durante dichas operaciones, y sólo después de un período de curado de un mes se vincularon a sus soportes.

El empleo de la teoría de cargas compensadas y el cuidado puesto en la redacción del proyecto permitió controlar flechas y deformaciones en toda la estructura. El pretensado de las vigas empezó rebasando ligeramente el estado de equilibrio por peso propio, con lo que se obtuvo una flecha de aproximadamente $12 \mathrm{~mm}$. Los cables de pretensado se proyectaron para equilibrar el propio peso de las losas y, de tal forma, que cuando éstas fueron co locadas y las vigas postesas no resultase flecha o deformación alguna anormal. Corrientemente, se detecta después de tesar una ligera flecha que curva a las vigas y las separa de los entramados auxiliares que las sostienen. Las losas también fueron postesadas para equilibrar su propio peso, y de tal suerte que se obtuviesen placas elásticas sometidas a una compresión prácticamente uniforme y dispuestas para soportar cargas dinámicas. En todo el entramado estructural pudo observarse que tanto las vigas como las losas se comportaron de acuerdo con lo que de ellas se esperaba, sin que se presentase problema alguno en lo que respecta a flechas y a deformaciones.

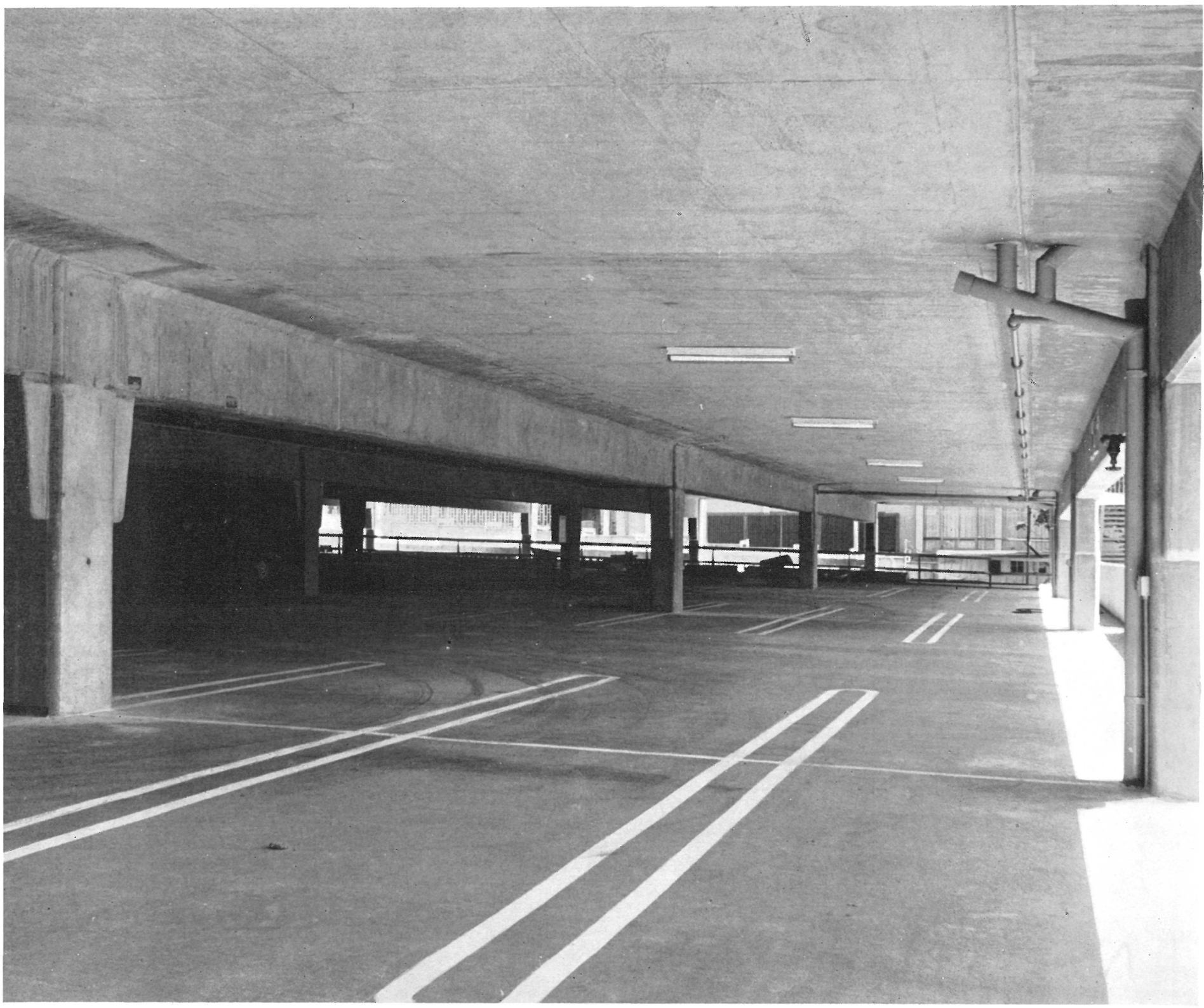




\section{Gamge poum I'Université de Californie}

T. Y. Lin et Félix Kulka, ingénieurs.

L'Université de Californie a fait construire, à Berkeley, un garage de deux étages, en béton précontraint, dont la parti. cularité est d'avoir utilisé la couverture pour soutenir une couche de terre de $45 \mathrm{~cm}$ d'épaisseur, couverte de gazon.

La structure portante se compose de poutres en treillis formant des travées de $7,30 \mathrm{~m}$ de portée et d'appuis séparés de $19 \mathrm{~m}$ maximum, les uns des autres. Les dalles des planchers ont done $7,30 \mathrm{~m}$ de portée, alors que celle des poutres est de 19 m maximum.

La dalle de couverture ayant été préconłrainte en deux directions normales entre elles et convenablement comprimée dans ces deux sens, l'apparition de fissures est pratiquement impossible. De plus, le tracé des câbles de la précontrainte sation.

Comme l'édifice est entouré, sur trois de ses façades, par autant de murs de soutènement, on a tiré profit de cette circonstance pour transmettre à ces murs les effets du vent, autant que sismiques, dans une direction, alors que ces effets, en direction normale à la première, sont transmis au terrain par l'intermédiaire des supports.

La plupart des éléments structuraux, à l'exception des dalles de planchers, ont été préfabriqués et précontraints en atelier, à environ $80 \mathrm{~km}$ du chantier.

Plusieurs de ces éléments ont été précontraints après leur mise en oeuvre.

\section{Garage for Galifomnia University}

T. Y. Lin \& Félix Kulka, engineers.

California University has constructed a two storey garage, with a prestressed concrete structure, at Berkeley.

A particular feature of this building is that on the roof a soil layer, $0.45 \mathrm{cms}$ in depth, has been laid, on which there grass lawn.

The structure consists of a framework of beams, of $7.30 \mathrm{~m}$ span, in one direction, and $19 \mathrm{~m}$ in the other: this being separatio

The roof slab has been prestressed in two mutually perpendicular directions, hence it is practically impossible for it to develop cracks. This arrangement renders the slab watertight and implies a saving in the cost of applying waterproofing treatment.

As the building is surrounded on three sides by three contention walls, advantage has been taken of this to transmit to these the horizontal thrusts due to windage and seismie forces.

Most of the structural elements, excepting the flooring, have been prestressed and prefabricated at a workshop $80 \mathrm{~km}$ away from the site.

Many of the constructional units have additional reinforcement, which can be post-tensioned after erection at the working site.

\section{Die Garmge der Universitüitin Kaliformien}

T. Y. Lin y Félix Kulga, Ingenieure.

Die Universität Berkeley in Kalifornien hat eine zweistöckige Garrage aus Spannbeton gebaut, bei der das Bemerkenswerte ist, dass man auf den oberen Teil des Daches eine 0,45 dicke Erdschicht aufgetragen und anschliessend Rasen darauf gepflanzt hat.

Die Struktur dieses Baus setzt sich aus einer Vielzahl von Trägern mit $7,30 \mathrm{~m}$ Spannweiten und aus Säulen mit $19 \mathrm{~m}$ Abständen zusammen. Die Deckenplatten überbrücken damit eíne Spannweite von 7,30 m, während die Träger eine Art Portale von $19 \mathrm{~m}$ maximaler Spannweite bilden.

Die Deckenplatten hat man in zwei senkrecht zu einander verlaufenden Richtungen unter sich vorgespannt, wodurch ein Auftreten von Rissen fast ausgeschlossen wurde. Diese Anordung der Vorspannkabel garantiert eine grosse Wasserundurchlässigkeit des Betons und hat auch wirtschaftliche Vorteile, da eine Isolierung nicht mehr notwendig war.

Da das Gebäude mit drei seiner Aussenwände an drei fremde Umfassungsmauern angrenzt, hat man daraus Nutzen gezogen und die Decken auf diesen Mauern vestrebt und damit auf sie die Wirkungen des Windes und von Erbeben in dieser
Richtung übertragen, während in der senkrecht dazu verlaufenden diese Wirkungen mit Hilfe der Säulen zur Erde geleitet werden.

Der grösse Teil der Bauteile mit Ausnahme der Deckenplatten, wurde in einem Werk $80 \mathrm{~km}$ von der Baustelle entfernt vorfabriziert und vorgespannt.

Viele der Bauelemente haben ausserdem noch eine zusätzliche Bewehrung bekommen und wurden nach ihrem Einbau noch nachgespannt. 